\title{
Some remarks on the sparse dominations for commutators of multi(sub)linear operator
}

Zhidan Wang ${ }^{1 *}$ (D)

\author{
"Correspondence: \\ zdwang@mail.bnu.edu.cn \\ ${ }^{1}$ School of Mathematical Sciences, \\ Beijing Normal University, \\ Laboratory of Mathematics and \\ Complex Systems, Ministry of \\ Education, Beijing, 100875, People's \\ Republic of China
}

\begin{abstract}
We establish pointwise sparse dominations for the iterated commutators of multi(sub)linear operators satisfying the $W_{q}$ condition. As consequences, we present some quantitative weighted estimates for the commutators. In addition, we also obtain the Fefferman-Stein inequality, the Coifman-Fefferman inequality, and the local decay estimates regarding the iterated commutators.
\end{abstract}

MSC: 42B20; 42B25

Keywords: Iterated commutators; Sparse dominations; Fefferman-Stein inequality; Coifman-Fefferman inequality; Local decay estimates

\section{Introduction}

Sparse domination is a relatively new tool to prove weighted estimates for singular integrals. The sparse method has been developed over the past five years by many researchers in harmonic analysis, with significant works by Lerner [1], Lacey [2], Conde-Alonso [3], and the references therein.

A collection $\mathcal{S}$ is an $\eta$-sparse family of cubes in $\mathbb{R}^{d}$ if for every $Q \in \mathcal{S}$ there exists $E_{Q} \subset Q$ such that $\left|E_{Q}\right| \geq \eta|Q|$ (here $0<\eta<1$ ), and $E_{Q} \cap E_{Q^{\prime}}=\varnothing$ when $Q \neq Q^{\prime}$. For an $\eta$-sparse collection of cubes $\mathcal{S}$, we use the notation

$$
\langle f\rangle_{p, Q}:=|Q|^{-\frac{1}{p}}\left\|f \chi_{Q}\right\|_{L^{p}}
$$

This averaging form is then easily controlled, facilitating the proof of weighted $A_{p}$-type estimates.

Lerner [1] proved a pointwise sparse domination for $\omega$ Calderón-Zygmund operators. The key role in his proof is played by the grand maximal operator

$$
\mathcal{M}_{T}(f)(x):=\sup _{Q \ni x} \operatorname{esssup}_{\xi \in Q}\left|T\left(f \mathbb{R}^{d} \backslash 3 Q\right)(\xi)\right|
$$

Later, $\mathrm{Li}$ [4] established sparse domination theorem for multilinear singular integral operators with the kernel satisfying the $L^{r}$-Hörmander condition. Cao and Yabuta [5] developed sparse dominations for the multilinear Littlewood-Paley operators with the same kernel

(c) The Author(s) 2021. This article is licensed under a Creative Commons Attribution 4.0 International License, which permits use sharing, adaptation, distribution and reproduction in any medium or format, as long as you give appropriate credit to the original author(s) and the source, provide a link to the Creative Commons licence, and indicate if changes were made. The images or other third party material in this article are included in the article's Creative Commons licence, unless indicated otherwise in a credit line to the material. If material is not included in the article's Creative Commons licence and your intended use is not permitted by statutory regulation or exceeds the permitted use, you will need to obtain permission directly from the copyright holder. To view a copy of this licence, visit http://creativecommons.org/licenses/by/4.0/ 
condition. Wen, Wu, and Xue [6] gave a sparse domination for the iterated commutators of multilinear pseudo-differential operators. Recently Lerner and Ombrosi [7] improved the results in [1] by weakening the assumption on $T$ and by replacing $\mathcal{M}_{T}(f)(x)$ with a more flexible operator. Motivated by the above works, the purpose of this paper is to establish a sparse domination for the iterated commutators of multi(sub)linear operator with weaker hypotheses than $[4,5]$.

Motivated by Lerner and Ombrosi [7], we assume that $T$ is an operator satisfying the following $W_{q}$ property instead of assuming $T$ is bounded from $L^{q} \times \cdots \times L^{q} \rightarrow L^{q / m, \infty}$ : there is a nonincreasing function $\psi_{T, q}$ such that, for any $f_{j} \in L^{q}(Q)$ with $j=1, \ldots, m$ and any cube $Q$,

$$
\left|\left\{x \in Q:\left|T\left(\vec{f} \chi_{Q}\right)(x)\right|>\psi_{T, q}(\lambda) \prod_{j=1}^{m}\left\langle f_{j}\right\rangle_{q, Q}\right\}\right| \leq \lambda|Q| \quad(0<\lambda<1) .
$$

It is easy to see that $L^{q} \times \cdots \times L^{q} \rightarrow L^{q / m, \infty}$ implies that $T$ satisfies the $W_{q}$ property with $\psi_{T, q}=\|T\|_{L^{q} \times \cdots \times L^{q} \rightarrow L^{q / m, \infty}} \lambda^{-m / q}$.

Let $\alpha>0$, we define

$$
\mathcal{M}_{T, \alpha}^{\sharp}(\vec{f})(x):=\sup _{Q \ni x} \operatorname{esssup} \xi_{\xi, \xi^{\prime} \in Q}\left|T\left(\vec{f} \chi_{\mathbb{R}^{d} \backslash \alpha Q}\right)(\xi)-T\left(\vec{f} \chi_{\mathbb{R}^{d} \backslash \alpha Q}\right)\left(\xi^{\prime}\right)\right|
$$

Given an operator $T, \alpha>0$, the iterated commutators of $T$ are defined by

$$
T_{\vec{b}}^{\ell}(\vec{f})(x)=\left[b_{1},\left[b_{2}, \ldots\left[b_{\ell}, T\right]_{\ell} \cdots\right]_{2}\right]_{1}(\vec{f})(x)
$$

where $\vec{b}=\vec{b}^{(\ell)}=\left(b_{1}, \ldots, b_{\ell}\right)(1 \leq \ell \leq m)$. Throughout this paper, $\tau_{m}=\{1, \ldots, m\}$. The symbol $|\tau|$ denotes the number of the elements in $\tau . \tau^{\prime}=\tau_{m} \backslash \tau$ is the complementary set.

Our main results of this paper are as follows.

Theorem 1.1 Assume that the multi(sub)linear integral T satisfies the $W_{q}$ condition and $\mathcal{M}_{T, \alpha}^{\sharp}$ is bounded from $L^{r} \times \cdots \times L^{r} \rightarrow L^{r / m, \infty}$ for some $\alpha \geq 3$. Let $1 \leq q, r<\infty$, and $s=$ $\max \{q, r\}$. Then, for any compactly supported functions $f_{i} \in L^{s}\left(\mathbb{R}^{d}\right), i=1, \ldots, m$, there exist $3^{d}$ sparse families $\mathcal{S}_{j}$ such that, for a.e. $x \in \mathbb{R}^{d}$,

$$
\begin{aligned}
& \left|T_{\vec{b}}^{\ell}(\vec{f})(x)\right| \\
& \quad \leq C \sum_{j=1}^{3^{d}} \sum_{\tau \subset \tau_{\ell}} \sum_{Q \in \mathcal{S}_{j}} \prod_{i \in \tau}\left\langle f_{i}\right\rangle_{s, Q}\left|b_{i}(x)-b_{i, Q}\right| \times \prod_{k \in \tau_{\ell} \backslash \tau}\left\langle\left(b_{k}-b_{k, Q}\right) f_{k}\right\rangle_{s, Q} \times \prod_{t \in \tau_{m} \backslash \tau_{\ell}}\left\langle f_{t}\right\rangle_{s, Q} \chi_{Q},
\end{aligned}
$$

where $C=c_{d, s, \alpha}\left(\psi_{T, q}\left(1 / 12 \cdot(2 \alpha)^{d}\right)+\left\|\mathcal{M}_{T, \alpha}^{\sharp}\right\|_{L^{r} \times \cdots \times L^{r} \rightarrow L^{r / m, \infty}}\right)$.

Remark 1.1 Our Theorem 1.1 is the commutators result of [7]. Compared with the hypotheses in $[4,5]$, the $\mathcal{M}_{T, \alpha}^{\sharp}$ makes our proof concise and clear. Further, the $W_{q}$ condition of $T$ is weaker than the assumption $L^{q} \times \cdots \times L^{q} \rightarrow L^{q / m, \infty}$ of $T$ in $[4,5]$. 
Let $1 \leq p_{1}, \ldots, p_{m} \leq \infty, \vec{w}=\left(w_{1}, \ldots, w_{m}\right)$, and each $w_{i}$ is a nonnegative function on $\mathbb{R}^{d}$. $\vec{w}$ is said to satisfy the following $A_{\vec{p} / r}$ condition if

$$
[\vec{w}]_{A_{\vec{p} / r}}:=\sup _{Q}\left(\frac{1}{|Q|} \int_{Q} v_{\vec{w}} \mathrm{~d} x\right) \prod_{k=1}^{m}\left(\frac{1}{|Q|} \int_{Q} w_{k}^{-\frac{r}{p_{i}-r}}(x) \mathrm{d} x\right)^{\frac{p\left(p_{i}-r\right)}{p_{i} r}},
$$

where $v_{\vec{w}}=\prod_{k=1}^{m} w_{k}^{p / p_{k}}$. When $r=1, A_{\vec{p} / r}$ is the $A_{\vec{p}}$ weight class defined by Lerner et al. [8].

With the pointwise sparse domination result, we can obtain the following quantitative weighted estimates and endpoint estimates from Sect. 3 in [5] immediately.

Corollary 1.2 Assume that the multi(sub)linear integral T satisfies the $W_{q}$ condition and $\mathcal{M}_{T, \alpha}^{\sharp}$ is bounded from $L^{r} \times \cdots \times L^{r} \rightarrow L^{r / m, \infty}$ for some $\alpha \geq 3$. Let $1 \leq q, r<\infty$, and $s=$ $\max \{q, r\}, \frac{1}{p}=\frac{1}{p_{1}}+\cdots+\frac{1}{p_{m}}$ with $1 \leq s<p_{i}<\infty$. Set $\sigma_{i}=w_{i}^{1-\left(p_{i} / s\right)^{\prime}}, i=1, \ldots$, m. Let $T_{\vec{b}}^{\ell}(\vec{f})$ be defined as (1.2).

(1) For $\vec{w} \in A_{\vec{p} / s}$, there exists a constant $C=C_{m, \vec{p}, d, s}$ such that

$$
\begin{aligned}
& \left\|T_{\vec{b}}^{\ell}(\vec{f})\right\|_{L^{p}\left(v_{\vec{w}}\right)} \\
& \quad \leq C[\vec{w}]_{A_{\vec{p} / s}}^{\max _{1 \leq i \leq m}\left\{1, \frac{1}{p}\left(\frac{p_{i}}{s}\right)^{\prime}\right\}}\left(\sum_{\tau \subset \tau_{\ell}}\left[v_{\vec{w}}\right]_{A_{\infty}}^{|\tau|} \prod_{j \in \tau_{\ell} \backslash \tau}\left[\sigma_{j}\right]_{A_{\infty}}\right) \prod_{i=1}^{\ell}\left\|b_{i}\right\|_{B M O} \prod_{j=1}^{m}\left\|f_{j}\right\|_{L^{p_{j}}\left(w_{j}\right)} .
\end{aligned}
$$

(2) If $\vec{w} \in A_{\overrightarrow{1}}$, then for any $\lambda>0$ and $\Phi_{s, \ell}=t^{s}\left(1+\log ^{+} t\right)^{s \ell}$ it holds that

$$
v_{\vec{w}}\left(\left\{x:\left|T_{\vec{b}}^{\ell}(\vec{f})\right|>\lambda^{m}\right\}\right) \leq C_{\vec{b}, s, \ell} \prod_{i=1}^{m}\left(\int_{\mathbb{R}^{d}} \Phi_{s, \ell}\left(\frac{\left|f_{i}(x)\right|}{\lambda}\right) w_{i} d x\right)^{1 / m} .
$$

We also obtain the local decay estimate, the Coifman-Fefferman inequality with $w \in A_{\infty}$ weight, and the Fefferman-Stein inequality with arbitrary weights regarding the iterated commutators. To the best knowledge of the author, these results are new for the iterated commutators of multi(sub)linear operator.

Theorem 1.3 Assume that the multi(sub)linear integral $T$ satisfies the $W_{q}$ condition and $\mathcal{M}_{T, \alpha}^{\sharp}$ is bounded from $L^{r} \times \cdots \times L^{r} \rightarrow L^{r / m, \infty}$ for some $\alpha \geq 3$. Let $1 \leq q, r<\infty, s=\max \{q, r\}$, and $a>s$, then for any functions $\operatorname{supp} f_{i} \subset Q, i=1, \ldots, m$, there exist constants $\alpha_{d, m}$ and $c_{d, m}$ such that

$$
\left|\left\{x \in Q: \frac{\left|T_{b}^{\ell}(\vec{f})\right|}{M_{a}(\vec{f})}>\lambda\right\}\right| \leq \alpha_{d, m} e^{-2 c_{d, m} \frac{\lambda}{\prod_{i \in \tau_{\ell}}\left\|b_{i}\right\|_{B M O O^{c}}}}|Q|, \quad \lambda>0 .
$$

Theorem 1.4 Assume that the multi(sub)linear integral $T$ satisfies the $W_{q}$ condition and $\mathcal{M}_{T, \alpha}^{\sharp}$ is bounded from $L^{r} \times \cdots \times L^{r} \rightarrow L^{r / m, \infty}$ for some $\alpha \geq 3$. Let $1 \leq q, r<\infty, s=\max \{q, r\}$, and $a>s$, then for any $1 \leq p<\infty$ and any weight $w \in A_{\infty}$,

$$
\left\|T_{b}^{\ell}(\vec{f})\right\|_{L^{p}(w)} \leq c_{d, m} \sum_{\tau \subset \tau_{\ell}}[w]_{A_{\infty}}^{|\tau|} \prod_{i \in \tau_{\ell}}\left\|b_{i}\right\|_{B M O}\left\|M_{a}(\vec{f})\right\|_{L^{p}(w)} .
$$


Theorem 1.5 Assume that the multi(sub)linear integral $T$ satisfies the $W_{q}$ condition and $\mathcal{M}_{T, \alpha}^{\sharp}$ is bounded from $L^{r} \times \cdots \times L^{r} \rightarrow L^{r / m, \infty}$ for some $\alpha \geq 3$. Let $1 \leq q, r<\infty$, the exponents $\frac{1}{p}=\frac{1}{p_{1}}+\cdots+\frac{1}{p_{m}}$ with $p>s$ and $1<p_{1}, \ldots, p_{m}<\infty$, then for all weights $\vec{w}=\left(w_{1}, \ldots, w_{m}\right), v_{\vec{w}}=\prod_{i=1}^{m} w_{i}^{p / p_{i}}$,

$$
\left\|T_{b}^{\ell}(\vec{f})\right\|_{L^{p}\left(v_{\vec{w}}\right)} \lesssim \prod_{i=1}^{m}\left\|f_{i}\right\|_{L^{p_{i}\left(M w_{i}\right)}}
$$

The article is organized as follows. Section 2 contains some definitions and main lemmas. The proof of theorems is given in Sect. 3. We present some variations of Theorem 1.1 in Sect. 4.

\section{Definitions and main lemmas}

We begin by introducing some definitions and notations.

Lemma 2.1 (Generalized Hölder inequality, [9]) Let $\Phi_{0}, \Phi_{1}, \ldots, \Phi_{k}$ be Young functions. If

$$
\Phi_{1}^{-1}(t) \Phi_{2}^{-1}(t) \cdots \Phi_{k}^{-1}(t) \leq \kappa \Phi_{0}^{-1}(t)
$$

then the following inequality holds:

$$
\left\|f_{1} f_{2} \cdots f_{k}\right\|_{\Phi_{0}, Q} \leq \kappa\left\|f_{1}\right\|_{\Phi_{1}, Q}\left\|f_{2}\right\|_{\Phi_{2}, Q} \cdots\left\|f_{k}\right\|_{\Phi_{k}, Q}
$$

for all functions $f_{1}, \ldots, f_{m}$ and all cubes $Q$.

In particular, if $\sum_{i=1}^{k} \frac{1}{s_{i}}=\frac{1}{s}$ with each $s_{i} \geq 1$, then it holds that

$$
\frac{1}{Q} \int_{Q}\left|f_{1} f_{2} \cdots f_{k} g\right| \leq C_{s}\left\|f_{1}\right\|_{\exp L^{s_{1}, Q}}\left\|f_{2}\right\|_{\exp L^{s_{2}, Q}} \cdots\left\|f_{k}\right\|_{\exp L^{s_{k}, Q}}\|g\|_{L(\log L)^{\frac{1}{s}, Q}} .
$$

We introduce the weighted maximal operator and the multi(sub)linear maximal operator which will be used in the proof of our theorem. Let $w$ be a weight and $a \geq 1$,

$$
M_{w} f(x)=\sup _{Q \ni x} \frac{1}{w(Q)} \int_{Q}|f| w d y, \quad M_{a}(\vec{f})=\sup _{Q \ni x} \prod_{i=1}^{m}\left(\frac{1}{|Q|} \int_{Q}\left|f_{i}\right|^{a} d y_{i}\right)^{\frac{1}{a}} .
$$

Lemma 2.2 ([5]) Let $s>1, t>0$, and $w \in A_{\infty}$, then there holds that

$$
\|f w\|_{L(\log L)^{t}, Q} \lesssim[w]_{A_{\infty}}^{t} \inf _{x \in Q} M_{w}\left(|f|^{s}\right)^{1 / s}\langle w\rangle_{Q} .
$$

Definition 2.1 The sidelength of $Q$ is denoted by $\ell(Q)$. Given a cube $Q_{0} \subset \mathbb{R}^{d}$, let $\mathcal{D}\left(Q_{0}\right)$ denote the generation of $Q_{0}$, that is, the cubes obtained by repeated subdivision of $Q_{0}$. Given dyadic grids $\mathcal{D}$, for any $j \in \mathbb{Z}$, the set $\mathcal{D}_{j}=\left\{Q \in \mathcal{D}, \ell(Q)=2^{j}\right\}$ forms a partition of $\mathbb{R}^{d}$. 


\section{Proof of theorems}

Now we devote to proving Theorem 1.1 with the case $\ell=m$. The other cases are similar so we omit their proof here. The basic idea of our proof is borrowed from Sect. 3 in [5], but the definition of $W_{q}$ and $\mathcal{M}_{T, \alpha}^{\sharp}$ makes our proof more convenient than [5].

Proof of Theorem 1.1 Fix a cube $Q_{0} \in \mathbb{R}^{d}$ and let $Q_{0}^{*}=\alpha Q_{0}$. Set

$$
\widetilde{M}_{T}(\vec{f})=\max \left(|T(\vec{f})|, \mathcal{M}_{T, \alpha}^{\sharp}(\vec{f})\right) .
$$

We define the set $E$ as follows:

$$
E=\left\{x \in Q_{0}: \max \left(\frac{M_{s}\left(\vec{f} \chi_{Q_{0}^{*}}\right)(x)}{c}, \frac{\tilde{M}_{T}(\vec{f})}{A}\right)>\prod_{j=1}^{m}\left\langle f_{j}\right\rangle_{s, Q_{0}^{*}}\right\} .
$$

By the weak endpoint estimates of $M_{s}(\vec{f})$ and (1.1), we can choose $c=c_{d, s, \alpha}$ and $A=$ $2 \psi_{T, q}\left(1 / 12 \cdot(2 \alpha)^{d}\right)+\left\|\mathcal{M}_{T, \alpha}^{\sharp}\right\|_{L^{r} \rightarrow L^{r, \infty}}$ such that $|E| \leq \frac{1}{2^{d+2}}\left|Q_{0}\right|$. Then, applying the local Calderón-Zygmund decomposition to $\chi_{E}$ on $Q_{0}$ at $\lambda=\frac{1}{2^{d+1}}$, we can get a family of pairwisely disjoint cubes $\left\{P_{l}\right\} \subset \mathcal{D}\left(Q_{0}\right)$ such that

$$
\frac{1}{2^{d+1}\left|P_{l}\right|}<\left|E \cap P_{l}\right| \leq \frac{1}{2}\left|P_{l}\right|, \quad\left|E \backslash \bigcup_{l} P_{l}\right|=0 .
$$

It is easy to have that $\sum_{l}\left|P_{l}\right| \leq \frac{1}{2}\left|Q_{0}\right|$ and $P_{l} \bigcap E^{c} \neq \emptyset$.

$$
\begin{aligned}
& \left|T_{\vec{b}}\left(\vec{f} \chi_{Q_{0}^{*}}\right)(x)\right| \chi_{Q_{0}}(x) \\
& \quad \leq\left|T_{\vec{b}}\left(\vec{f} \chi_{Q_{0}^{*}}\right)(x)\right| \chi_{Q_{0} \backslash \cup_{l} P_{l}}+\sum_{l}\left|T_{\vec{b}}\left(\vec{f} \chi_{Q_{0}^{*} \backslash P_{l}^{*}}\right)(x)\right| \chi_{P_{l}}+\sum_{l}\left|T_{\vec{b}}\left(\vec{f} \chi_{P_{l}^{*}}\right)(x)\right| \chi_{P_{l}} \\
& \quad:=I+I I+I I I .
\end{aligned}
$$

From [10, Remark 5.1], there exist $3^{d}$ dyadic lattices $\mathcal{D}_{j}$ such that for every cube $Q \subset \mathbb{R}^{d}$ we can find a cube $R_{Q} \in \mathcal{D}_{j}$ satisfying $3 Q \subset R_{Q}$ and $\left|R_{Q}\right| \leq 9^{n}|Q|$. Note that $\prod_{i=1}^{m}\left(b_{i}(x)-\right.$ $\left.b_{i}\left(y_{i}\right)\right)=\sum_{\tau \subset \tau_{m}} \prod_{i \in \tau}\left(b_{i}(x)-b_{i, R_{Q_{0}}}\right) \prod_{j \in \tau^{\prime}}\left(b_{j, R_{Q_{0}}}-b_{j}\left(y_{j}\right)\right)$. Then we can write

$$
T_{\vec{b}}(\vec{f})(x)=\sum_{\tau \subset \tau_{m}} \prod_{i \in \tau}\left(b_{i}(x)-b_{i, R_{Q_{0}}}\right) T\left(\prod_{i \in \tau} f_{i}\left(y_{i}\right) \prod_{j \in \tau^{\prime}}\left(b_{j, R_{Q_{0}}}-b_{j}\left(y_{j}\right)\right) f_{j}\left(y_{j}\right)\right)(x) .
$$

Since $\left|E \backslash \bigcup_{l} P_{l}\right|=0$, it follows that

$$
I \lesssim A \sum_{\tau \subset \tau_{m}} \prod_{i \in \tau}\left\langle f_{i}\right\rangle_{s, Q_{0}^{*}}\left|b_{i}(x)-b_{i, R_{Q_{0}}}\right| \prod_{j \in \tau^{\prime}}\left\langle\left(b_{j}-b_{j, R_{Q_{0}}}\right) f_{j}\right\rangle_{s, Q_{0}^{*}} \chi_{Q_{0}} .
$$

Now we calculate $I I$ in (3.1). For $x \in P_{l}$ and $x^{\prime} \in P_{l} \backslash E$,

$$
\begin{aligned}
& \sum_{l}\left|T_{\vec{b}}\left(\vec{f} \chi_{Q_{0}^{*} \backslash P_{l}^{*}}\right)(x)\right| \chi_{P_{l}} \\
& \quad \leq \sum_{l} \sum_{\tau \subset \tau_{m}} \prod_{i \in \tau}\left|b_{i}(x)-b_{i, R_{Q_{0}}}\right|\left|T\left(\prod_{i \in \tau} f_{i} \chi_{Q_{0}^{*} \backslash P_{l}^{*}} \prod_{j \in \tau^{\prime}}\left(b_{j, R_{Q_{0}}}-b_{j}\right) f_{j} \chi_{Q_{0}^{*} \backslash P_{l}^{*}}\right)(x)\right| .
\end{aligned}
$$


Denoting $\vec{g} \chi_{Q_{0}^{*} \backslash P_{l}^{*}}(x)=\prod_{i \in \tau} f_{i} \chi_{Q_{0}^{*} \backslash P_{l}^{*}} \prod_{j \in \tau^{\prime}}\left(b_{j, R_{Q_{0}}}-b_{j}\right) f_{j} \chi_{Q_{0}^{*} \backslash P_{l}^{*}}(x)$, we can write

$$
\begin{aligned}
\left|T \vec{g} \chi_{Q_{0}^{*} \backslash P_{l}^{*}}(x)\right| & \leq \inf _{P_{l}} \mathcal{M}_{T, \alpha}^{\sharp}\left(\vec{g} \chi_{Q_{0}^{*} \backslash P_{l}^{*}}\right)(x)+\left|T\left(\vec{g} \chi_{Q_{0}^{*} \backslash P_{l}^{*}}\right)\left(x^{\prime}\right)\right| \\
& \lesssim A \prod_{i \in \tau}\left\langle f_{i}\right\rangle_{s, Q_{0}^{*}} \prod_{j \in \tau^{\prime}}\left\langle\left(b_{j}-b_{j, R_{Q}}\right) f_{j}\right\rangle_{s, Q_{0}^{*}}+\left|T\left(\vec{g} \chi_{Q_{0}^{*}}\right)\left(x^{\prime}\right)\right|+\left|T\left(\vec{g} \chi_{P_{l}^{*}}\right)\left(x^{\prime}\right)\right| \\
& \lesssim 2 A \prod_{i \in \tau}\left\langle f_{i}\right\rangle_{s, Q_{0}^{*}} \prod_{j \in \tau^{\prime}}\left\langle\left(b_{j}-b_{j, R_{Q_{0}}}\right) f_{j}\right\rangle_{s, Q_{0}^{*}}+\left|T\left(\vec{g} \chi_{P_{l}^{*}}\right)\left(x^{\prime}\right)\right| .
\end{aligned}
$$

By $\left|P_{l} \backslash E\right| \geq \frac{\left|P_{l}\right|}{2}$ and $\left|\left\{x \in P_{l}:\left|T\left(\vec{f} \chi_{P_{l}^{*}}\right)\left(x^{\prime}\right)\right|>A \prod_{j=1}^{m}\left\langle f_{j}\right\rangle_{s, P_{l}^{*}}\right\}\right| \leq \frac{\left|P_{l}\right|}{2^{d+2}}$, we can get that

$$
\inf _{P_{l} \backslash E}\left|T\left(\vec{g} \chi_{P_{l}^{*}}\right)\right| \leq A \prod_{j=1}^{m}\left\langle g_{j}\right\rangle_{s, P_{l}^{*}} \leq c A \prod_{j=1}^{m}\left\langle g_{j}\right\rangle_{s, Q_{0}^{*}}
$$

This allows us to continue (3.3) with

$$
\left|T \vec{g} \chi_{Q_{0}^{*} \backslash P_{l}^{*}}(x)\right| \lesssim(2+c) A \prod_{i \in \tau}\left\langle f_{i}\right\rangle_{s, Q_{0}^{*}} \prod_{j \in \tau^{\prime}}\left\langle\left(b_{j}-b_{j, R_{0}}\right) f_{j}\right\rangle_{s, Q_{0}^{*}} .
$$

Therefore

$$
I I \lesssim(2+c) A \sum_{\tau \subset \tau_{m}} \prod_{i \in \tau}\left\langle f_{i}\right\rangle_{s, Q_{0}^{*}}\left|b_{i}(x)-b_{i, R_{Q_{0}}}\right| \prod_{j \in \tau^{\prime}}\left\langle\left(b_{j}-b_{j, R_{Q_{0}}}\right) f_{j}\right\rangle_{s, Q_{0}^{*}} \chi_{Q_{0}} .
$$

III in (3.1) is the term we need. Combining (3.2) with (3.4), it follows that

$$
\begin{aligned}
\left|T_{\vec{b}}\left(\vec{f} \chi_{Q_{0}^{*}}\right)\right| \chi_{Q_{0}}(x) \lesssim & (3+c) A \sum_{\tau \subset \tau_{m}} \prod_{i \in \tau}\left\langle f_{i}\right\rangle_{s, Q_{0}^{*}}\left|b_{i}(x)-b_{i, R_{Q_{0}}}\right| \prod_{j \in \tau^{\prime}}\left\langle\left(b_{j}-b_{j, R_{Q_{0}}}\right) f_{j}\right\rangle_{s, Q_{0}^{*}} \chi_{Q_{0}} \\
& +\sum_{l}\left|T_{\vec{b}}\left(\vec{f} \chi_{P_{l}^{*}}\right)(x)\right| \chi_{P_{l}} .
\end{aligned}
$$

Integrating the above estimates, we can get a $\frac{1}{2}$-sparse family $\mathcal{F} \subset \mathcal{D}\left(Q_{0}\right)$ such that for a.e. $x \in Q_{0}$

$$
\left|T_{\vec{b}}\left(\vec{f} \chi_{Q_{0}^{*}}\right)\right| \lesssim(3+c) A \sum_{Q \in \mathcal{F}} \sum_{\tau \subset \tau_{m}} \prod_{i \in \tau}\left\langle f_{i}\right\rangle_{s, Q^{*}}\left|b_{i}(x)-b_{i, R_{Q}}\right| \prod_{j \in \tau^{\prime}}\left\langle\left(b_{j}-b_{j, R_{Q}}\right) f_{j}\right\rangle_{s, Q^{*}} \chi_{Q} \cdot
$$

The remaining procedure which transfers local setting $Q_{0}$ to global setting $\mathbb{R}^{d}$ can be referred to Sect. 4.2 in [5]. We omit the details to avoid redundancy. The proof of Theorem 1.1 is finished now.

Proof of Theorem 1.3 Let $\ell=m$. The proof of other cases is similar. Assume $\operatorname{supp} f_{i} \subset Q_{0}$, $i=1, \ldots, m$, and denote

$$
\mathcal{A}_{\mathcal{F}, \tau}(\vec{b}, \vec{f}):=\sum_{Q \in \mathcal{F}} \prod_{i \in \tau}\left\langle f_{i}\right\rangle_{s, Q^{*}}\left|b_{i}(x)-b_{i, R_{Q}}\right| \prod_{j \in \tau^{\prime}}\left\langle\left(b_{j}-b_{j, R_{Q}}\right) f_{j}\right\rangle_{s, Q^{*}} \chi_{Q}
$$


It is straightforward to see that we can replace $b_{R_{Q}}$ with $b_{Q^{*}}$ in $\mathcal{A}_{\mathcal{F}, \tau}(\vec{b}, \vec{f})$. Then, by (3.5), we can get a $\frac{1}{2}$-sparse family $\mathcal{F} \subset \mathcal{D}\left(Q_{0}\right)$ such that, for almost every $x \in Q_{0}$,

$$
\left|T_{\vec{b}}(\vec{f})\right|=\left|T_{\vec{b}}\left(\vec{f} \chi_{Q_{0}^{*}}\right)\right| \lesssim \sum_{\tau \subset \tau_{m}} \mathcal{A}_{\mathcal{F}, \tau}(\vec{b}, \vec{f})
$$

First we observe that $\left|b_{i}(x)-b_{i, Q^{*}}\right| \lesssim\left\|b_{i}\right\|_{B M O}+\left|b_{i}(x)-b_{i, Q}\right|$. From [11, Lemma 5.1], we can construct a sparse family $\tilde{\mathcal{F}} \subset \mathcal{D}\left(Q_{0}\right)$ such that, for every $Q \in \mathcal{F} \subset \tilde{\mathcal{F}}$,

$$
\left|b_{i}(x)-b_{i, Q}\right| \lesssim c_{d} \sum_{P \in \tilde{\mathcal{F}}, P \subseteq Q}\left(\frac{1}{|P|} \int_{P}\left|b_{i}-b_{i, P}\right| d x\right) \chi_{P}(x) \lesssim c_{d}\left\|b_{i}\right\|_{B M O} \sum_{P \in \tilde{\mathcal{F}}, P \subseteq Q_{0}} \chi_{P}(x) .
$$

For any $a>s$, Lemma 2.1 gives that

$$
\left\langle\left(b_{j}-b_{j, Q^{*}}\right) f_{j}\right\rangle_{s, Q^{*}} \lesssim\left\|b_{j}\right\|_{B M O}\left\langle f_{j}\right\rangle_{a, Q^{*}} .
$$

Now we can write

$$
\begin{aligned}
& \mathcal{A}_{\mathcal{F}, \tau}(\vec{b}, \vec{f}) \\
& \quad \lesssim \sum_{Q \in \mathcal{F}} \prod_{i=1}^{m}\left\langle f_{i}\right\rangle_{a, Q^{*}}\left\|b_{i}\right\|_{B M O} \chi_{Q}+c_{d, m} \sum_{Q \in \mathcal{F}} \prod_{i=1}^{m}\left\langle f_{i}\right\rangle_{a, Q^{*}}\left\|b_{i}\right\|_{B M O} \sum_{P \in \tilde{\mathcal{F}}, P \subseteq Q_{0}} \chi_{P}(x) \chi_{Q} .
\end{aligned}
$$

Hence, it is straightforward to have that

$$
\begin{aligned}
\frac{\mathcal{A}_{\mathcal{F}, \tau}(\vec{b}, \vec{f})(x)}{M_{a} \vec{f}(x)} & \leq c_{d, m} \sum_{Q \in \mathcal{F}} \prod_{i=1}^{m}\left\|b_{i}\right\|_{B M O} \chi_{Q}+c_{d, m} \sum_{Q \in \mathcal{F}} \prod_{i=1}^{m}\left\|b_{i}\right\|_{B M O} \sum_{P \in \tilde{\mathcal{F}}, P \subseteq Q_{0}} \chi_{P}(x) \chi_{Q} \\
& \leq c_{d, m} \prod_{i=1}^{m}\left\|b_{i}\right\|_{B M O} \sum_{P \in \tilde{\mathcal{F}}, P \subseteq Q_{0}} \chi_{P}(x) .
\end{aligned}
$$

[12, Lemma 2.1] gives that

$$
\begin{aligned}
\left|\left\{x \in Q_{0}: \frac{\mathcal{A}_{\mathcal{F}, \tau}(\vec{b}, \vec{f})}{M_{a}(\vec{f})}>\lambda\right\}\right| & \leq\left|\left\{x \in Q_{0}: \frac{\sum_{P \in \tilde{\mathcal{F}}, P \subseteq Q_{0}} \chi_{P}(x)}{M_{a} \vec{f}}>\frac{\lambda}{c_{d, m} \prod_{i=1}^{m}\left\|b_{i}\right\|_{B M O} c_{T}}\right\}\right| \\
& \leq c e^{-\alpha_{d, m} \frac{\lambda}{\prod_{i=1}^{m}\left\|b_{i}\right\|_{B M O^{c} T}}}\left|Q_{0}\right| .
\end{aligned}
$$

This finishes the proof of Theorem 1.3.

Proof of Theorem 1.4 Let $\ell=m$. From Theorem 1.1, it only needs to control $\mathcal{A}_{\mathcal{S}, \tau}(\vec{b}, \vec{f})$. By duality, there exists a nonnegative function $g \in L^{p^{\prime}}(w)$ satisfying $\|g\|_{L^{p^{\prime}}(w)}=1$. Then we can write

$$
\begin{aligned}
& \int_{\mathbb{R}^{n}} \mathcal{A}_{\mathcal{S}, \tau}(\vec{b}, \vec{f}) g w d x \\
& =\sum_{Q \in \mathcal{S}} \prod_{i \in \tau}\left\langle f_{i}\right\rangle_{s, Q} \int_{Q} \prod_{i \in \tau}\left|b_{i}(x)-b_{i, Q}\right| g(x) w(x) d x \prod_{j \in \tau^{\prime}}\left\langle\left(b_{j}-b_{j, Q}\right) f_{j}\right\rangle_{s, Q}
\end{aligned}
$$




$$
\begin{aligned}
& \lesssim \sum_{Q \in \mathcal{S}} \prod_{i \in \tau}\left\langle f_{i}\right\rangle_{s, Q}|Q|\left\|\left(b_{i}-b_{i, Q}\right)\right\|_{\exp L, Q}\|g w\|_{L(\log L)^{|\tau|}, Q} \prod_{j \in \tau^{\prime}}\left\|b_{j}\right\|_{B M O}\left\langle f_{j}\right\rangle_{a, Q} \\
& \lesssim \prod_{i=1}^{m}\left\|b_{i}\right\|_{\mathrm{BMO}}\left\langle f_{i}\right\rangle_{a, Q} \sum_{Q \in \mathcal{S}}[w]_{A_{\infty}}^{|\tau|} \inf _{x \in Q} M_{w}\left(|g|^{q}\right)(x)^{1 / q} w(Q),
\end{aligned}
$$

where $s<a$. The second inequality follows from Lemma 2.1, and we have used Lemma 2.2 in the last inequality. By [13], we know $M_{w}\left(|g|^{q}\right)(x)^{1 / q}$ is $L^{p^{\prime}}\left(w_{j}\right)$ bounded when $1<q<p^{\prime}$. We can continue writing above display as

$$
\begin{aligned}
& \int_{\mathbb{R}^{n}} \mathcal{A}_{\mathcal{S}, \tau}(\vec{b}, \vec{f}) g w d x \\
& \quad \lesssim[w]_{A_{\infty}}^{|\tau|} \prod_{i=1}^{m}\left\|b_{i}\right\|_{\mathrm{BMO}} \sum_{Q \in \mathcal{S}} \int_{Q} M_{a}(\vec{f})(x) M_{w}\left(|g|^{q}\right)(x)^{1 / q} w d x \\
& \quad \lesssim[w]_{A_{\infty}}^{|\tau|} \prod_{i=1}^{m}\left\|b_{i}\right\|_{\mathrm{BMO}} \int_{\mathbb{R}^{d}} M_{a}(\vec{f})(x) M_{w}\left(|g|^{q}\right)(x)^{1 / q} w d x \\
& \quad \lesssim[w]_{A_{\infty}}^{|\tau|} \prod_{i=1}^{m}\left\|b_{i}\right\|_{\mathrm{BMO}}\left\|M_{a}(\vec{f})(x)\right\|_{L^{p}(w)}\left\|M_{w}\left(|g|^{q}\right)(x)^{1 / q}\right\|_{L^{p^{\prime}}(w)} \\
& \quad \lesssim[w]_{A_{\infty}}^{|\tau|} \prod_{i=1}^{m}\left\|b_{i}\right\|_{\mathrm{BMO}}\left\|M_{a}(\vec{f})(x)\right\|_{L^{p}(w)}\|g\|_{L^{p^{\prime}(w)}}
\end{aligned}
$$

where $1<q<p^{\prime}$. This finishes the proof of Theorem 1.4.

Proof of Theorem 1.5. The basic idea of our proof is borrowed from Sect. 4.3 in [14] or [15]. From Theorem 1.1, it is enough to control $\mathcal{A}_{\mathcal{S}, \tau}(\vec{b}, \vec{f})$. Let $\ell=m$.

$$
\left\|\mathcal{A}_{\mathcal{S}, \tau}(\vec{b}, \vec{f})\right\|_{L^{p}\left(v_{\vec{w}}\right)}^{p} \leq \sum_{Q \in \mathcal{S}} \prod_{i \in \tau}\left\langle f_{i}\right\rangle_{s, Q}^{p} \int_{Q} \prod_{i \in \tau}\left|b_{i}(x)-b_{i, Q}\right|^{p} v_{w}(x) d x \prod_{j \in \tau^{\prime}}\left\langle\left(b_{j}-b_{j, Q}\right) f_{j}\right\rangle_{s, Q}^{p}
$$

We denote $v_{i}(x):=M w_{i}(x)$, then it is easy to see that $\left\langle w_{i}\right\rangle_{Q} \leq v_{i}(x)$ for any cube $Q$ containing $x$. We can choose constants $a, b$ with $s<a<p<b$. [11, Lemma 5.1] gives us a sparse family $\tilde{\mathcal{S}}$ such that, for every $Q \in \mathcal{S} \subset \tilde{\mathcal{S}}$, it holds that

$$
\left|b_{i}(x)-b_{i, Q}\right| \lesssim\left\|b_{i}\right\|_{B M O} \sum_{P \in \tilde{\mathcal{S}}, P \subseteq Q} \chi_{P}(x)
$$

By Lemma 2.1, it holds that

$$
\begin{aligned}
\left\|\mathcal{A}_{\mathcal{S}}(\vec{b}, \vec{f})\right\|_{L^{p}\left(v_{\vec{w}}\right)}^{p} & \\
& \lesssim \sum_{Q \in \mathcal{S}} \prod_{i \in \tau}\left\langle f_{i}\right\rangle_{s, Q}^{p}\left\|b_{i}\right\|_{B M O}^{p} \sum_{P \in \tilde{\mathcal{S}}, P \subseteq Q} \chi_{P}(x) \prod_{j \in \tau^{\prime}}\left\langle\left(b_{j}-b_{j, Q}\right) f_{j}\right\rangle_{s, Q}^{p} v_{\vec{w}}(P) \\
& \lesssim \sum_{Q \in \mathcal{S}} \prod_{i \in \tau}\left\langle f_{i}\right\rangle_{s, Q}^{p}\left\|b_{i}\right\|_{B M O}^{p} \sum_{P \in \tilde{\mathcal{S}}, P \subseteq Q} \prod_{j \in \tau^{\prime}}\left\|b_{j}\right\|_{\mathrm{BMO}}^{p}\left\langle f_{j}\right\rangle_{a, Q}^{p}\left\langle v_{\vec{w}}\right\rangle_{P}|P|
\end{aligned}
$$




$$
\begin{aligned}
& \lesssim \sum_{Q \in \mathcal{S}} \prod_{i=1}^{m}\left\langle f_{i} v_{i}^{\frac{1}{p_{i}}}\right\rangle_{p, Q}^{p}\left\langle\left. v_{i}^{-\frac{1}{p_{i}}}\right|_{b, Q} ^{p}\left\|b_{i}\right\|_{B M O}^{p} \sum_{P \in \tilde{\mathcal{S}}, P \subseteq Q}\left\langle v_{\vec{w}}\right\rangle_{P}|P|\right. \\
& \lesssim \sum_{Q \in \mathcal{S}} \prod_{i=1}^{m}\left\langle f_{i} \frac{1}{\bar{p}_{i}}\right\rangle_{p, Q}^{p}\left\|b_{i}\right\|_{B M O}^{p} \sum_{P \in \tilde{\mathcal{S}}, P \subseteq Q}\left\langle w_{i}\right\rangle_{P}^{-\frac{p}{p_{i}}}\left\langle v_{\vec{w}}\right\rangle_{P}|P| .
\end{aligned}
$$

The sparseness property of collection $\mathcal{S}, \tilde{\mathcal{S}}$ allows us to continue with

$$
\begin{aligned}
\left\|\mathcal{A}_{\mathcal{S}}(\vec{b}, \vec{f})\right\|_{L^{p}\left(v_{\vec{w}}\right)}^{p} & \lesssim \sum_{Q \in \mathcal{S}} \prod_{i=1}^{m}\left\langle\left. f_{i} v_{i}^{\frac{1}{p_{i}}}\right|_{p, Q} ^{p}\left\|b_{i}\right\|_{B M O}^{p} \sum_{P \in \tilde{\mathcal{S}}, P \subseteq Q}\left|E_{P}\right|\right. \\
& \lesssim \sum_{Q \in \mathcal{S}} \prod_{i=1}^{m}\left(\inf _{Q} M_{p}\left(f_{i} v_{i}^{\frac{1}{p_{i}}}\right)\right)^{p}\left\|b_{i}\right\|_{B M O}^{p}|Q| \\
& \lesssim \int_{\mathbb{R}^{d}} \prod_{i=1}^{m}\left(M_{p}\left(f_{i} v_{i}^{\frac{1}{p_{i}}}\right)\right)^{p} d x \prod_{i=1}^{m}\left\|b_{i}\right\|_{B M O}^{p} \\
& \lesssim \prod_{i=1}^{m}\left\|b_{i}\right\|_{B M O}^{p}\left\|f_{i}\right\|_{L^{p_{i}}\left(M w_{i}\right)}^{p}
\end{aligned}
$$

This finishes the proof of Theorem (1.3).

\section{Variations}

Let us introduce some notions first. $T$ is a Calderón-Zygmund operator defined as

$$
T \vec{f}(x)=\int_{\left(\mathbb{R}^{d}\right)^{m}} K(x, \vec{y}) \prod_{j=1}^{m} f_{j}\left(y_{j}\right) d \vec{y}, \quad x \notin \bigcap_{j=1}^{m} \operatorname{supp} f_{j} .
$$

Definition 4.1 ([4]) Let $1 \leq r<\infty, r^{\prime}=\frac{r}{r-1}$. The kernel $K(x, \vec{y})$ is called to satisfy the multilinear $L^{r}$-Hörmander condition if

$$
K_{r}:=\sup _{Q} \sup _{x, z \in \frac{1}{2} Q} \sum_{k=1}^{\infty}\left|2^{k} Q\right|^{\frac{m}{r}}\left(\int_{\left(2^{k} Q\right)^{m} \backslash\left(2^{k-1} Q\right)^{m}}|K(x, \vec{y})-K(z, \vec{y})|^{r^{\prime}} d \vec{y}\right)^{\frac{1}{r^{\prime}}}<\infty .
$$

When $r=1$, it should be understood as

$$
K_{1}:=\sup _{Q} \sup _{x, z \in \frac{1}{2} Q} \sum_{k=1}^{\infty}\left|2^{k} Q\right|^{\frac{m}{r}} \underset{\vec{y} \in\left(2^{k} Q\right)^{m} \backslash\left(2^{k-1} Q\right)^{m}}{\operatorname{esssup}}|K(x, \vec{y})-K(z, \vec{y})|<\infty .
$$

We first show that the multilinear $L^{r}$-Hörmander condition implies that $\mathcal{M}_{T, \alpha}^{\sharp}(\vec{f})$ is bounded from $L^{r} \times \cdots \times L^{r} \rightarrow L^{r / m, \infty}$ with $1 \leq r<\infty$.

Lemma 4.1 Suppose $1 \leq r<\infty, \alpha \geq 3$. T is an operator whose kernel satisfies the bilinear $L^{r}$-Hörmander condition. Then $\mathcal{M}_{T, \alpha}^{\sharp}(\vec{f})$ is bounded from $L^{r} \times \cdots \times L^{r} \rightarrow L^{r / m, \infty}$. More specifically, for any $x \in \mathbb{R}^{d}$, it holds that

$$
\mathcal{M}_{T, \alpha}^{\sharp}(\vec{f})(x) \leq K_{r} \mathcal{M}_{r}(\vec{f})(x),
$$

where $\mathcal{M}_{r}(\vec{f})(x)=\mathrm{M}\left(|\vec{f}|^{r}\right)^{\frac{1}{r}}$ and $\mathrm{M}$ is the Hardy-Littlewood maximal function. 
Proof Let $\xi, \xi^{\prime} \in Q$.

$$
\begin{aligned}
& \left|T\left(\vec{f} \chi_{\mathbb{R}^{d} \backslash \alpha Q}\right)(\xi)-T\left(\vec{f} \chi_{\mathbb{R}^{d} \backslash \alpha Q}\right)\left(\xi^{\prime}\right)\right| \\
& \quad \leq\left|\int_{\left(\mathbb{R}^{d}\right)^{m} \backslash(\alpha Q)^{m}}\left(K(\xi, \vec{y})-K\left(\xi^{\prime}, \vec{y}\right)\right) \prod_{j=1}^{m} f_{j}\left(y_{j}\right) d \vec{y}\right| \\
& \quad \leq \sum_{k=1}^{\infty}\left|2^{k} Q\right|^{\frac{m}{r}}\left(\int_{\left(2^{k} Q\right)^{m} \backslash\left(2^{k-1} Q\right)^{m}}\left|K(\xi, \vec{y})-K\left(\xi^{\prime}, \vec{y}\right)\right|^{r^{\prime}} d \vec{y}\right)^{\frac{1}{r^{\prime}}}\left(\frac{1}{\left|2^{k} Q\right|^{m}} \int_{\left(2^{k} Q\right)^{m}}|\vec{f}|^{r} d \vec{y}\right)^{\frac{1}{r}} \\
& \quad \leq K_{r} \mathcal{M}_{r}(\vec{f})(x) .
\end{aligned}
$$

Since $\mathcal{M}_{r}(\vec{f})(x)$ is bounded from $L^{r} \times \cdots \times L^{r} \rightarrow L^{r / m, \infty}$, we can get the weak type estimates of $\mathcal{M}_{T, \alpha}^{\sharp}(\vec{f})$.

Theorem 4.2 Let the multi(sub)linear integral T satisfy the $W_{q}$ condition and the kernel satisfy the multilinear $L^{r}$-Hörmander condition with $1 \leq q, r<\infty$, then all the estimates in Sect. 1 hold for this operator $T$.

We give another variation of Theorem 1.1. In this case, we do not need to assume that $T$ is a multi(sub)linear operator. Given an operator $T(\vec{f})$ and $\alpha>0$, we define

$$
\mathcal{M}_{T, \alpha}^{\sharp}(\vec{f})(x):=\sup _{Q \ni x} \operatorname{esssup}\left|\left(T \overrightarrow{\xi^{\prime} \in Q} \mid \vec{f}-T\left(\vec{f} \chi_{\alpha Q}\right)\right)(\xi)-\left(T \vec{f}-T\left(\vec{f} \chi_{\alpha Q}\right)\right)\left(\xi^{\prime}\right)\right| .
$$

Theorem 4.3 Let $1 \leq q, r<\infty, \alpha \geq 3$. There are nonincreasing functions $\psi$ and $\phi$ such that, for any cube $Q$,

$$
\begin{aligned}
& \left|\left\{x \in Q:\left|T\left(\vec{f} \chi_{Q}\right)(x)\right|>\psi(\lambda) \prod_{j=1}^{m}\left\langle f_{j}\right\rangle_{q, Q}\right\}\right| \leq \lambda|Q| \quad(0<\lambda<1) ; \\
& \left.\left|\left\{x \in Q: \mid \mathcal{M}_{T, Q}^{\#} \vec{f} \chi_{Q}\right)(x)\right|>\phi(\lambda) \prod_{j=1}^{m}\left\langle f_{j}\right\rangle_{r, Q}\right\}|\leq \lambda| Q \mid \quad(0<\lambda<1) .
\end{aligned}
$$

Then all the estimates in Sect. 1 hold for this operator $T$.

Remark 4.2 The assumption of $\mathcal{M}_{T, \alpha}^{\sharp} \vec{f}$ satisfying the $W_{r}$ condition allows us not to prove Lemma 4.1. In addition, we should use $T_{\vec{b}}\left(\vec{f} \chi_{Q_{0}}\right)(x)-T_{\vec{b}}\left(\vec{f} \chi_{P_{l}^{*}}\right)(x)$ instead of $T_{\vec{b}}\left(\vec{f} \chi_{Q_{0}^{*} \backslash P_{l}^{*}}\right)(x)$ to avoid using the multi(sub)linear property.

Acknowledgements

The author wants to express thanks to Qingying Xue for his valuable suggestions on improving this work.

Funding

Not applicable.

Availability of data and materials

Not applicable. 
Authors' contributions

The authors worked jointly in drafting and approving the final manuscript. All authors read and approved the final manuscript.

\section{Publisher's Note}

Springer Nature remains neutral with regard to jurisdictional claims in published maps and institutional affiliations.

Received: 2 January 2021 Accepted: 5 March 2021 Published online: 12 March 2021

\section{References}

1. Lerner, A.K.: On pointwise estimates involving sparse operators. N.Y. J. Math. 22, 341-349 (2016)

2. Lacey, M.T.: An elementary proof of the A2 bound. Isr. J. Math. 217, 181-195 (2017)

3. Conde-Alonso, J.M., Culiuc, A., Di Plinio, F., Ou, Y.: A sparse domination principle for rough singular integrals. Anal. PDE 10(5), 1255-1284 (2017). https://doi.org/10.2140/apde.2017.10.1255

4. Li, K.: Sparse domination theorem for multilinear singular integral operators with $L^{r}$-Hörmander condition. Mich. Math. J. 67(2), 253-265 (2018). https://doi.org/10.1307/mmj/1516330973

5. Cao, M., Yabuta, K.: The multilinear Littlewood-Paley operators with minimal regularity conditions. J. Fourier Anal. Appl. 25(3), 1203-1247 (2019)

6. Wen, Y., Wu, H., Xue, Q.: A note on multilinear pseudo-differential operators and iterated commutators. Bull. Korean Math. Soc. 57(4), 851-864 (2020). https://doi.org/10.4134/BKMS.b190532

7. Lerner, A.K., Ombrosi, S.: Some remarks on the pointwise sparse domination. J. Geom. Anal. 30(1), 1011-1027 (2020) https://doi.org/10.1007/s12220-019-00172-9

8. Lerner, A.K., Ombrosi, S., Pérez, C., Torres, R.H., Trujillo-González, R.: New maximal functions and multiple weights for the multilinear Calderón-Zygmund theory. Adv. Math. 220(4), 1222-1264 (2009) https://doi.org/10.1016/j.aim.2008.10.014

9. Pérez, C., Trujillo-González, R.: Sharp weighted estimates for multilinear commutators. J. Lond. Math. Soc. (2) 65(3) 672-692 (2002). https://doi.org/10.1112/S0024610702003174

10. Ibañez-Firnkorn, G.H., Rivera-Ríos, I.P.: Sparse and weighted estimates for generalized Hörmander operators and commutators. Monatshefte Math. 191(1), 125-173 (2020)

11. Lerner, A.K., Ombrosi, S., Rivera-Ríos, I.P.: On pointwise and weighted estimates for commutators of Calderón-Zygmund operators. Adv. Math. 319,153-181 (2017). https://doi.org/10.1016/j.aim.2017.08.022

12. Ortiz-Caraballo, C., Pérez, C., Rela, E.: Exponential decay estimates for singular integral operators. Math. Ann. 357(4), 1217-1243 (2013). https://doi.org/10.1007/s00208-013-0940-3

13. Culiuc, A., Di Plinio, F., Ou, Y.: Domination of multilinear singular integrals by positive sparse forms. J. Lond. Math. Soc 98(2), 369-392 (2018). https://doi.org/10.1112/jlms.12139

14. Cao, M., Hormozi, M., Ibañez-Firnkorn, G., Rivera-Ríos, I.P., Si, Z., Yabuta, K.: Weak and strong type estimates for the multilinear Littlewood-Paley operators (2020) Available at https://arxiv.org/pdf/2009.13814.pdf

15. Cao, M., Si, Z., Zhang, J.: Weak and strong types estimates for square functions associated with operators (2020) Available at https://arxiv.org/pdf/2011.11420v1.pdf

\section{Submit your manuscript to a SpringerOpen ${ }^{\circ}$ journal and benefit from:}

- Convenient online submission

- Rigorous peer review

- Open access: articles freely available online

- High visibility within the field

- Retaining the copyright to your article

Submit your next manuscript at $>$ springeropen.com 